\title{
SOME MATHEMATICAL PROBLEMS ARISING IN THE STUDY OF QUASICRYSTALS
}

\author{
F. GÄHLER \\ Theoretische Physik, ETH-Hönggerberg, CH-8093 Zürich, \\ switzerland
}

RESUME. Après une revue sommaire de la méthode de projection quelques propriétés des quasi-cristaux sont derivées pour des structures obtenues à l'aide de cette méthode. On demontre en particulier la connection entre les spectres de Fourier et les classes d'équivalence induites par un isomorphisme local. On propose que l'entropie résiduelle est zéro a $T=0$. Enfin certaines propriétés associées à la self-similarité sont discutées.

\begin{abstract}
After briefly reviewing the projection method, some properties of quasicrystal structures obtained by projection are discussed. In particular, the connection between the Fourier spectra and the local isomorphism class of a quasicrystal is worked out. It is argued that quasicrystals have no residual entropy at $T=0$. Finally, certain self-similarity properties of quasicrystals are discussed.
\end{abstract}

\section{Introduction}

The discovery of an icosahedral phase of $A l-M n$ alloy by Shechtman et al. [1] has initiated much activity in the field, both experimental and theoretical. Such quasiperiodic structures have been termed quasicrystals [2]. Meanwhile, several other quasicrystals have been described, some with different rotational symmetry $[3,4]$, and many with different chemical composition (for references, see these proceedings). On the theoretical side, the interest has concentrated on structures obtained by the strip or projection method [5], which is based on de Bruijn's work [6] on Penrose tilings [7]. These projected patterns can be obtained also with a different but equivalent algorithm [8] (the grid method), which goes back to de Bruijn [6] too, and which has advantages when one wants to generate quasicrystal patterns on a computer.

In this paper we discuss some mathematical aspects of the models obtained by the projection method. After briefly reviewing this method in section 2, we calculate the Fourier spectra of 
quasicrystals in section 3. These Fourier transforms are formally given by sums of $\delta$-functions whose positions fill reciprocal space densely. We then point out the connection between the Fourier transform and the concept of local isomorphism. Two patterns are called locally isomorphic if and only if every finite portion of one occurs also in the other one, and vice versa.

It is shown that the Fourier transforms of locally isomorphic quasicrystal patterns have the same positions and intensities of the $\delta$-peaks, whereas patterns which are not locally isomorphic have Fourier transforms that differ in the peak intensities. Jocal isomorphism is a standard concept in tiling theory [7]. It has been introduced into the field of quasicrystals by Levine and Steinhardt [9], who were also the first to discuss the connection with the Fourier transform of the Ammann quasilattice associated with a quasicrystal. The Fourier transform of a quasicrystal however can be rather different from that of its Ammann quasilattice.

In section 4 we argue that physical structures which are based on locally isomorphic patterns should be expected to be energetically degenerate. We then derive bounds on the number of locally isomorphic patterns that differ within a given finite region. More precisely, we obtain bounds on the number of neighbourhoods of radius $R$ a given yertex can have within a fixed local isomorphism class. It is shown that this number grows at most as a power of $R$. This implies that quasicrystals have no residual entropy at $T=0$.

Finally, in section 5 we argue that a certain type of self-similarity occurs typically in many quasicrystals. It is explained how this property can be used in the analysis of diffraction patterns of quasicrystals.

\section{The projecton method}

In this section we briefly review the projection method. More details can be found in $[5,10,11]$. Consider an n-dim. lattice

$$
L=\left\{\mathrm{x} \in E^{n} \mid \mathrm{x}=\sum_{i=1}^{n} k_{i} \mathrm{e}_{i}, k_{i} \in Z\right\}
$$

where $\left\{\mathbf{e}_{i}\right\}$ is a basis of $E^{n}$. Choose an $n$-dim. subspace $E^{\|} \subset E^{n}$ and let $E^{\perp}$ be its orthogonal complement, so that $E^{n}=E^{H} \oplus E^{\perp}$. Denote by $e_{i}^{i}$ and $e_{i}^{\perp}$ the projection of $e_{i}$ onto $E^{4}$ and $E^{\perp}$ respectively. In the same way decompose $x \in E^{n 2}$ as $x=x_{\|}+x_{\perp}$.

For every vector $y \in E^{n}$ we define a strip

$$
S_{\gamma}=\left\{\mathrm{x} \in E^{\mathrm{n}} \mid \mathbf{x}=\mathbf{x}_{\mathbb{1}}+\gamma-\sum_{i=1}^{n} \alpha_{i} \mathbf{e}_{i}^{\perp} ; \mathbf{x}_{\| 1} \in E^{\mathrm{il}}, \alpha_{i} \in[0,1]\right\}
$$


Note that the cross section of $S_{\gamma}, S_{\gamma} \cap E^{\perp}$, is equal to the projection of an elementary n-cell of $L$ onto $E^{\perp}$.

Then the quasicrystal pattern is given by

$$
Q_{\gamma}=\sum_{x \in \mathcal{L} \cap s_{\gamma}} \delta\left(\mathbf{x}_{\mathrm{H}}\right)
$$

i.e. it is obtained by projecting all vertices in $L \cap S_{\gamma}$ onto $E^{\text {n. }}$.

$Q_{\gamma}$ has a translation symmetry $\mathbf{n}$ if and only if $\mathbf{n}$ is a lattice vector of $L$ contained in $E^{\| \prime}$.

\section{Fourier spectra and local isomorphism}

In the following we present a formal calculation of the Fourier spectra of $Q_{\gamma}$ and then comment on how this can be made rigorous. We use the notation of Zia and Dallas [12].

Using the density of lattice points of $L$,

$$
\rho_{L}(\mathbf{x})=\sum_{\mathbf{x} \in Z} \delta(\mathbf{x})
$$

we can write

$$
Q_{7}\left(x_{\| 1}\right)=\int d x_{\perp} \chi_{7}\left(x_{\perp}\right) p_{L}\left(x_{\|}, x_{\perp}\right),
$$

where $\chi_{\gamma}$ is the characteristic function of the strip $S_{\gamma}$. Since the integral of a function is its Fourier transform at 0 , we have

$$
Q_{\gamma}\left(\mathbf{x}_{\| 1}\right)=\int d \mathbf{k}_{\perp} \tilde{\chi}_{\gamma}\left(-\mathbf{k}_{\perp}\right) \tilde{\rho}_{L}\left(\mathbf{x}_{i 1}, \mathbf{k}_{\perp}\right)
$$

and thus

$$
\begin{aligned}
\tilde{Q}_{\gamma}\left(\mathbf{k}_{11}\right) & =\int d \mathbf{k}_{\perp} \tilde{\chi}_{\mathcal{T}}\left(-\mathbf{k}_{\perp}\right) \tilde{\tilde{\rho}}_{\mathcal{L}}\left(\mathbf{k}_{\mathfrak{l}}, \mathbf{k}_{\perp}\right) \\
& =\int d \mathbf{k}_{\perp} p_{\tilde{L}}(\mathbf{k})
\end{aligned}
$$

where $\tilde{\chi}_{\gamma}$ is the Fourier transform of $\chi_{\gamma}$ and $\tilde{\tilde{p}}=\rho_{\tilde{L}}$ is the density of points in the reciprocal lattice $\tilde{L}$. Therefore $\tilde{Q}_{\gamma}$ is obtained by multiplying every vertex of $\tilde{L}$ by $\tilde{X}_{\gamma}\left(-\mathbf{k}_{\perp}\right)$ and then projecting onto $\widetilde{E}^{\prime \prime}$. In this way one obtains a sum of $\delta$-functions which typically are dense in $\widetilde{E}^{\prime \prime}$.

Since $\chi_{\gamma}$ is discontinuous and thus $\tilde{\chi}_{\gamma}$ decays only slowly, the intensities of the $\delta$-peaks even in a finite region of $\tilde{E}^{\prime \prime}$ are not summable and therefore the above calculation is only formal. However $Q_{\gamma}$ and thus $\chi_{\gamma}$ can be defined as tempered distributions. Using a sequence of smooth approximations $\chi_{\gamma}^{n}$ of $\chi_{\gamma}$, we obtain a sequence of tempered distributions $\tilde{Q}_{\gamma}^{n}$ converging to $\widetilde{Q}_{\gamma}$ (in the sense of distributions), and each element of this sequence is a (locally summable) sum of 
6-functions. This regularisation procedure should be kept in mind in the rest of this section. A similar approach to a rigorous theory of Fourier spectra of quasicrystals has been proposed by de Bruijn [13].

Let us now go back to the patterns in direct space. As the subscript indicates, $Q_{\gamma}\left(x_{11}\right)$ depends on $\gamma$. However, two patterns with labels $\gamma$ and $\boldsymbol{\gamma}^{\prime}$ differ by just a translation if and only if either $\Delta \gamma=\gamma-\gamma^{\prime}$ is a lattice vector of $L$ or $\Delta \gamma=\Delta \gamma_{\|}$. Therefore two patterns are translation equivalent iff

$$
\Delta y \in\left\{\mathbf{x} \in E^{\perp} \mid \mathbf{x}=\sum_{i=1}^{n} k_{i} \mathbf{e}_{i}^{\perp}, k_{i} \in Z\right\} \equiv K .
$$

Let us consider the closure $K$ of $K$. It is easy to see that $K$ has the structure

$$
K=\Lambda^{m} \times E^{m^{\prime}}, \quad m+m^{\prime}=n-d
$$

where $\Lambda^{m}$ is some $m$-dim. lattice.

If $\Delta \gamma_{\perp} \in K$, then there exists a sequence of patterns $Q_{\gamma^{\prime \prime}}$ such that $Q_{\gamma^{n}}$ is a translation of $Q_{\gamma}$ and $\gamma_{\perp}^{n}$ converges to $\gamma_{\perp}$. On the other hand, such a sequence exists only if $\Delta \gamma_{\perp} \in K$. Now observe that two patterns with parameters $\gamma$ and $\gamma^{\prime}$ agree in regions of radius $R$ centered at $\gamma_{\|}$and $\gamma_{11}^{\prime}$ respectively, if only $\left|\Delta \gamma_{\perp}\right|$ is small enough. Therefore we have proved that every finite portion of a pattem $Q_{\gamma}$ occurs also in $Q_{\gamma^{\prime}}$ and vice versa, if and only if $\Delta \gamma_{\perp} \in \mathcal{K}$. By the same reasoning we find that every finite portion of a pattern occurs even infinitely often, with a density which is the same for all patterns with $\Delta \gamma_{\perp} \in R$.

DEFinITION: Two patterns are called locally isomorphic if and only if every finite portion of one occurs also in the other one, and vice versa.

THEOREM. Two patterns $Q_{\gamma}$ and $Q_{\gamma^{\prime}}$ are locally isomorphic if and only if $\Delta \gamma_{\perp} \in K$.

We now relate these properties to the Fourier transforms. Suppose there is a lattice vector $\sum m_{i} \tilde{e}_{i}$ in the reciprocal lattice which is contained in $\tilde{E}^{\perp}$. Then there is an infinity of points in $\tilde{L}$ which is projected onto the same spot. When we shift the strip by changing $\gamma$, each vertex of $\tilde{L}$ picks up a phase and under projection there may occur interferences which could alter the intensity of a peak. This is the case if and only if $\Delta \gamma$ has a non-vanishing component in the direction of some lattice vector contained in $\tilde{E}^{\perp}$. Otherwise all vertices that are projected onto the same spot pick up the same phase. 
THEORBM. The Fourier transforms of two quasicrystal patterns agree in intensity if and only if the two patterns are locally isomorphic.

Proof: We have to show that the span of the lattice vectors of $\widetilde{L}$ contained in $\widetilde{E}^{\perp}$ is identical to the span of the lattice vectors of $\Lambda^{m}$ in (3.6). Suppose $\mathbf{u}=\sum m_{i} \tilde{\mathrm{e}}_{i}=\sum m_{i} \tilde{\mathrm{e}}_{i}^{1}$, i.e. $\sum m_{i} \tilde{\mathrm{e}}_{i}^{\|}=0$. Then we have

$$
2 \pi m_{i}=\mathbf{e}_{i} \cdot \sum m_{j} \tilde{e}_{j}=e_{j}^{+} \cdot \sum m_{j} \tilde{e}_{j}^{+}
$$

and therefore $n \in \Lambda^{m}$, since the $e_{i}^{\frac{1}{i}}$ are rationally dependent in the direction of $\mathbf{u}$. On the other hand $\Lambda^{m}$ is spanned by vectors $v_{\perp} \in E^{\perp}$ which satisfy $y_{\perp} \cdot e_{i}=2 \pi m_{i}, m_{i} \in Z$. But the same holds true also for $\mathbf{w}=\sum m_{j} \tilde{\mathbf{e}}_{j}$, and since $\left\{\mathbf{e}_{i}\right\}$ is a basis $\mathbf{w}$ must coincide with $\mathbf{v}_{1}$, and therefore w $\in \tilde{E}^{\perp}$.

This result can be understood also in a different way. Instead of projecting the vertices inside the strip one can decorate each vertex of $L$ with a piece of hyperplane parallel to $E^{\perp}$ and with the shape of the cross section of the strip, and then take the intersection of $E^{\text {u }}$ with this decorated periodic structure [14]. This has the advantage that one can use different strips for different types of vertices at the same time.

If there are lattice vectors $\mathrm{v} \in E^{\perp}$ we can project the lattice $L$ onto a lower dimensional one $L^{\prime}$, which is decorated with a basis. Each type of atom has then to be decorated with its own type of strip [14]. A change of local isomorphism class amounts in a change of the decoration of $L^{\prime}$, and it is therefore not surprising that this induces a change in the intensities of the diffraction pattern.

\section{Estimate on the number of ground states}

Suppose a quasicrystal pattern describes the ground state of a physical system with finite range interactions. Since in two locally isomorphic patterns every finite portion occurs in both of them with the same density, a finite range interaction cannot distinguish the two patterns. Therefore we conclude that all locally isomorphic patterns (uncoutably many !) describe energetically degenerate (ground) states. In order to obtain a better measure on the number of ground states we first estimate this number for a finite volume $V$ and then study how fast this number grows with $V$ in the thermodynamic limit.

More precisely, we estimate the number $N(R)$ of different neighbourhoods of radius $R$ a vertex of a quasicrystal pattern can have. This is done as follows. Choose a vertex $x \in L$ and a ball 
$B(R) \subset E^{\|}$of radius $R$, centered at $\mathbf{x}^{H}$. Now move the strip $S$ through $E^{\perp}$, but subject to the conditions that i) $x \in S$ and ii) the local isomorphism class is preserved, and count the number of patterns distinct in $B(R)$ we pick up in this way. The vertices $y \in L$ which are projected onto a vertex of at least one of these patterns have the property that i) $y_{\|} \in B(R)$ and ii) $y_{\perp} \in C \subset E^{\perp}$, where $C$ is some compact domain which is independent of $R$. Therefore the number of these vertices, $n(R)$, can be bounded by $n(R) \leq$ const. $\cdot V(R)$, where $V(R)$ is the volume of $B(R)$.

The translations of the strip which preserve the local isomorphism class consist of $m^{\prime}$ independent continuous translations and a lattice $\Delta^{m}$ of discrete translations (compare section 3 ). The condition that $\mathrm{x} \in S$ restricts this to continuous translations in a compact domain and finitely many discrete translations.

Under a continuous translation some new vertices enter the strip, others leave the strip. However, they enter and leave the strip in a fixed order and therefore under a continuous translation we pick up at most $2 n(R)$ patterns. Since there are $m^{\prime}$ independent continuous translations we obtain a bound

$$
N(R) \leq \text { const. } \cdot V(R)^{m^{\prime}}
$$

Note that the finitely many discrete transiations can affect only the constant in (4.1). Moreover our proof holds true also in the case where the cross section of the strip is any compact region in $E^{\perp}$.

The bound (4.1) implies the absence of residual (zero temperature) entropy density since

$$
s(T=0)=k \cdot \lim _{R \rightarrow \infty} \frac{1}{V(R)} \ln N(R)=0 .
$$

This result can be understood intuitively also from the fact that the whole quasicrystal pattern is completely specified by finitely many real parameters (the vector $\gamma$ ).

\section{Selfaimilarity properties}

A quasicrystal pattern may be called self-similar if there exists a systematic procedure (inflation) of erasing a subset of the vertices such that one obtains a pattern in the same local isomorphism class, but on a larger scale. Moreover the inverse procedure (deflation) is required to exist too.

In the following we argue that this kind of self-similarity is a typical phenomenon which is likely to occur whenever the rotation symmetry of a quasicrystal is large enough. A typical scenario might be the following one. 
Suppose there exists a symmetric invertible linear mapping $M: E^{n} \rightarrow E^{n}$ which maps $L$ onto $L$. Therefore the matrix of $M$ with respect to any lattice basis is contained in $G L_{n}(Z)$. This implies also that $\operatorname{det} M= \pm 1$. Assume moreover that $M$ leaves both $E^{\| 1}$ and $E^{\perp}$ invariant, $M\left|E^{\| l}=c \cdot 1_{E \| l}, c>1, M\right| E^{\perp} \leq 1_{E \perp}$, and that for any lattice vector $v \in E^{\perp}$ we have $M v=$ v. Such a mapping $M$-if it exists-has the property that it streches directions parallel to the strip, while directions perpendicular to the strip shrink (with the exception of those which are lattice directions). Therefore $M$ maps the strip onto a subset of the strip. Under this inflation transformation only the vertices in this subset survive. The mapping $M^{-1}$ enlarges the strip and provides thus the deflation procedure. On the other hand, such a quasicrystal pattern is completely specified by the grid vectors and the tiling vectors (see Ref. 8 ). These however have just grown by a constant factor $c$. Therefore, after inflation we have a pattern of the same type, but on a larger scale. The property $M v=v$ guarantees that one stays in the same local isomorphism class.

In this generality it is certainly difficult to decide whether such a mapping $M$ exists. Let us therefore consider a more specific case. Assume that $L$ has a point group $G$ which acts with an $R$-reducible representation on $L$, and suppose that we project on an irreducible representation space of $G$. If $G$ is large enough this imposes strong arithmetic conditions on the orientation with which $E^{\text {al }}$ is embedded relative to the lattice. It seems that these conditions facilitate the search for an appropriate $M$ considerably. For the icosahedral case such a mapping has been given by Elser [15]. For 2-dim. patterns with $C_{n}$ symmetry obtained by projection from an n-dim. cubic lattice (see e.g. [8]) the author has determined such mappings for $n=5,7,8,10,12$, with streching factors $\tau^{2}, 1+2 \cos \alpha-2 \cos 3 \alpha, 1+\sqrt{2}, \tau^{2}, 2+\sqrt{3}$ respectively ( $\tau$ is the golden ratio, $\alpha=\frac{2 \pi}{T}$ ). In some of the $2 \mathrm{~d}$ examples, the streching factor is negative, which induces a rotation by $\pi$. Instead, we could use the square of $M$ and therefore also the square of the streching factor.

It is not known whether a crystallographically forbidden point group acting on $E^{\text {" enforces }}$ self-similarity, but the experience with a few examples indicates that self-similarity is a typical phenomenon. A systematic study of a few important cases has been started also by Ostlund and Wright [16]. In contrast to our results these authors find no evidence for self-similarity in the 7-fold symmetric case.

We should point out that the self-similarity described here is a weaker one than that of Penrose patterns. In general, unlike in the Penrose case, it is not possible to give local rules for which vertices have to be added under deflation. To decide this one would have to know the whole pattern. Nevertheless, self-similarity is an important tool in the study of quasicrystals. For 
instance, the same analysis can be applied to the reciprocal lattice and this allows one then to derive scaling relations for the intensities of the peaks in much the same way as Elser [15] did in the icosahedral case.

Acknowledgements: The author would like to thank J. Rhyner for extensive discussions, as well as J. Fröhlich for various suggestions and continuous encouragement.

\section{References}

[1] D. Shechtman et al., Phys. Rev. Lett. 53 (1984) 1951-1953

[2] D. Levine and P.J. Steinhardt, Phys. Rev. Lett. 53 (1984) 2477-2480

[3] T. Ishimasa, H.U. Nissen and Y. Fukano, Phys. Rev. Lett. 55 (1985) 511-513

[4] L. Bendersky, Phys. Rev. Lett. 55 (1985) 1461-1463

[5] P. Kramer and R. Neri, Acta Cryst. A 40 (1984) 580-587

M. Duneau and A. Katz, Phys. Rev. Lett. 54 (1985) 2688-2691

V. Elser, Acta Cryst. A 42 (1986) 36-43

P.A.Kalugin et al., JETP Lett. 41 (1985) 145-149

[6] N.G. de Brujin, Ned. Akad. Wetensch. Proc. Ser. A 43 (1981) 39-66

[7] M. Gardner, Sci. Am., Jan. 1977, 110-121

[8] F. Gähler and J. Rhyner, J. Phys. A 19 (1986) 267-277

[9] D. Levine and P.J. Steinhardt, to appear in Phys. Rev. B (1986)

[10] A. Katz and M. Duneau, J. Physique 47 (1986) 181-196

[11] P. Kramer, Z. Naturforsch. 40a (1985) 775-788

[12] R.K.P. Zia and W.J. Dallas, J. Phys. A 18 (1985) L341-L345

[13] N.G. de Bruijn, preprint

[14] T. Janssen, to appear in Acta Cryst. $A$ (1986), and these proceedings

[15] V. Elser, Phys. Rev. B 32 (1985) 4892-4898

[16] S. Ostlund and D.C. Wright, Univ. Pennsylvania preprint 
COMMENTS AFTER THE F. GÄHLER, TALKS :

N. RIVIER - Continuous transformations (tunneling modes) between two different, non locally isomorphic configurations are physically observable in glasses ${ }^{1}$.

The structure of a covalent glass can be modelled by a continuous random network (CRN = regular graph), made of 4-bonded tetrapods, with slight, random bending of the bonds. There appear rings with odd number of bonds, threaded through by uninterrupted lines ("disclinations" characterized by oddness).

Configurations are described by transporting the tetrapod about a ring (Burgers). They are labelled by classes of the permutation group $\mathrm{S}_{4}$ (permutations of the feet of the tetrapod) : even permutations for even rings, odd permutations for odd rings. There are 2 odd classes of $S_{4}$, hence two configurations per odd line, which are not locally isomorphic ${ }^{2}$.

Tunneling between these two configurations does occur, and has been observed experimentally, most directly by (acoustic) echo techniques ${ }^{1}$.

1. C.F. W.A. PHILlIPS, Amorphous Solids, Springer 1981

2. N. RIVIER and H. GILCHRIST, J. Non-Cryst. Solids 75 (1985), 259, and to be published. 